\title{
Internal Bernstein Functions and Lévy-Laplace exponents
}

\author{
Kholoud Basalim ${ }^{1}$, Safa Bridaa ${ }^{2}$, and Wissem Jedidi ${ }^{3}$ \\ ${ }^{1}$ King Saud University \\ ${ }^{2}$ Universite de Tunis El Manar Faculte des Sciences de Tunis \\ ${ }^{3}$ University of Tunis El Manar Department of Mathematics
}

May 5, 2020

\begin{abstract}
Bertoin, Roynette et Yor (missing citation) described new connections between the class $\$ \backslash B d \$$ of $L \backslash$ 'evy-Laplace exponents $\$ \backslash \mathrm{Psi} \$$ (also called the class (sub)critical branching mechanism) and the class of Bernstein functions (\$BF $\$$ ) which are internal, i.e. those Bernstein functions $\$ \backslash$ phi $\$$ s.t. $\$ \backslash$ Psi $\backslash$ circ $\backslash$ phi $\$$ remains a Bernstein function for every $\$ \backslash$ Psi $\$$. We complete their work and illustrate how the class $\mathrm{f}$ internal function is rich from the stochastic point of view. It is well known that every $\$ \backslash$ phi $\backslash$ in $\backslash \mathrm{BF} \$$ corresponds univocally to: (i) a subordinator $\$\left\{\left(\mathrm{X}_{-} \mathrm{t}\right)\right\}_{-}\{\mathrm{t} \backslash$ geq 0$\} \$$ (or equivalently to transition semigroups $\$\left\{\backslash \operatorname{big}\left(\backslash \operatorname{pr}\left(X_{-} t \backslash \text { in } \mathrm{dx}\right) \backslash \text { big }\right)\right\}_{-}\{\mathrm{t} \backslash$ geq 0$\} \$$; (ii) a $\mathrm{L} \backslash$ 'evy measure $\$ \backslash \mathrm{mu} \$$ (which controls the jumps of the subordinator). It is also known that, on $\$ \backslash \mathrm{oi} \$$, the measure $\$ \backslash \operatorname{pr}\left(\mathrm{X}_{-} \mathrm{t} \backslash \mathrm{in} \mathrm{dx}\right) / \mathrm{t} \$$ converges vaguely to $\$ \backslash \mathrm{dd} \backslash \mathrm{delta}_{-}(\mathrm{dx})+\backslash \mathrm{mu}(\mathrm{dx}) \$$ as $\$ \mathrm{t} \backslash$ to $0 \$$, where $\$ \backslash \mathrm{dd} \$$ is the drift term, but rare are the situations where we can compare the transition semigroups with the L $\backslash$ 'evy measure. Our extensive investigations on the composition of $L \backslash$ 'evy-Laplace exponents $\$ \backslash P$ si $\$$ with Bernstein functions show, for instance, this remarkable facts: $\$ \backslash$ phi $\$$ is internal is equivalent to: (a) $\$ \backslash p h i \wedge 2 \backslash$ in $\backslash \mathrm{BF} \$$ or to (b) $\$ \mathrm{t} \backslash \mathrm{mu}(\mathrm{dx})-\backslash \mathrm{pr}\left(\mathrm{X} \_t \backslash\right.$ in $\mathrm{dx}) \$$ is a positive measure on $\$ \backslash \mathrm{oi} \$$. We also provide conditions on $\$ \backslash \mathrm{mu} \$$ insuring that $\$ \backslash$ phi $\$$ is internal. We also show $\mathrm{L} \backslash$ 'evy-Laplace exponents are closely connected to the class of Thorin Bernstein function and provide conditions on $\$ \backslash$ mu $\$$ insuring that $\$ \backslash$ phi $\$$ is internal.
\end{abstract}

\section{Hosted file}

Internality.pdf available at https://authorea.com/users/302383/articles/432454-internalbernstein-functions-and-1\%C3\%A9vy-laplace-exponents

\section{Hosted file}

Internality.tex available at https://authorea.com/users/302383/articles/432454-internalbernstein-functions-and-1\%C3\%A9vy-laplace-exponents

\section{References}

